\title{
Sintering analysis of sub-micron-sized nickel powders: Kinetic Monte Carlo simulation verified by FIB-SEM reconstruction
}

\author{
Shotaro Hara ${ }^{1,2}$, Akihiro Ohi ${ }^{1}$ and Naoki Shikazono ${ }^{1,2}$ \\ ${ }^{1}$ Institute of Industrial Science, The University of Tokyo, Komaba 4-6-1, Meguro-ku, Tokyo \\ 153-8505, Japan \\ ${ }^{2}$ JST CREST, Gobancho 7, Chiyoda-ku, Tokyo 102-0076, Japan
}

(Dated: **, 2014)

\begin{abstract}
Since sintering of sub-micron-sized particles is a critical phenomenon affecting the electrochemical performance and reliability of solid oxide fuel cell systems, a better understanding of this microstructure-related process is of great importance. In this study, we show that kinetic Potts Monte Carlo modeling is capable of quantitatively predicting the three-dimensional (3D) microstructure evolution over an entire stage of nickel sintering at the sub-micron scale. This is achieved through direct comparison of simulation results and 3D microstructural analysis using focused ion beam-scanning electron microscopy. We show that grain boundary diffusion is the dominant mechanism on densification, while surface diffusion has an impact on the coarsening during sub-micron scale sintering, only acting as one of the multiple mechanisms of sintering.
\end{abstract}

Keywords: Solid Oxide Fuel Cell; Sintering; Microstructure; Nickel; Monte Carlo Simulation; FIB-SEM

* Corresponding author: harasho@iis.u-tokyo.ac.jp

Tel \& Fax; +81-3-5452-6776

Address: Komaba 4-6-1, Meguro-ku, Tokyo 153-8505, Japan 


\section{Introduction}

Sintering of sub-micron-sized particles $(<1 \mu \mathrm{m})$ is a key technological phenomenon involved in solid oxide fuel cell (SOFC) systems, which are promising energy conversion devices because of their high efficiency and wide applications [1-3]. For example, the microstructure of SOFC electrodes, commonly fabricated via mixed powder sintering at the sub-micron scale, shows a strong correlation with their electrochemical performance [4-8]. In addition, microstructural changes in SOFC anodes associated with metallic particle sintering from exposure to high temperature leads to severe performance loss during operation [9-14]. Thus, to produce more reliable devices, a method that allows quantitative prediction of sintering behavior at the sub-micron scale is highly in demand.

Accurate prediction of microstructural evolution during sintering, even in single-phase systems, is a difficult task. This is because the response of sintering is deeply coupled with the three-dimensional (3D) complex geometries of powder compacts, such as the grain and pore size distributions, grain shape, relative density, and coordination number [15]. Among many types of numerical approaches [16-19], a mesoscale kinetic Potts Monte Carlo (KMC) method has the potential to overcome this difficulty [20-22]. This method is capable of capturing 3D microstructural evolution from the initial to the final stage of solid-state sintering, handling a few hundred non-spherical particles. More importantly, in conjunction with the quantitative assessment of 3D microstructure using X-ray tomography [23], it has been demonstrated that the numerical simulation is capable of reproducing the proper sintering trajectory for real copper powder of grain size over $40 \mu \mathrm{m}$ [20].

Motivated by these previous studies, we aim to construct a quantitative and predictive numerical model that allows us to understand microstructure evolution during sub-micron scale sintering. We achieve this goal by addressing the following issues. First, it should be noted that the 3D image-based techniques have their limits of resolution $[24,25]$. To quantify the 3D topologies of sub-micron-sized powder compacts, a technique that can achieve nanoscale resolution is essential. Second, from a physical viewpoint, the dominant mechanism governing mass flow during sintering is known to exhibit grain-size dependency. Indeed, surface diffusion becomes dominant for nanoscale sintering $(<50 \mathrm{~nm})$ [26, 27]. Conversely, grain-boundary diffusion has been considered to be the dominant mechanism 
when the grain size is greater than $10 \mu \mathrm{m}$ [28]. However, the underlying kinetics at the sub-micron scale are not yet completely understood.

In this study, we address nickel (Ni) sintering, which has been a significant durability issue in SOFC [8-14]. Quantitative topological changes during sintering of real powder compacts are measured using focused ion beam-scanning electron microscopy (FIB-SEM) [29]. This technique offers a great advantage in its available resolution, which is sufficient to capture the 3D geometric nature of sub-micron-sized powder compacts (typically $<1 / 10$ of the powder size). Simultaneously, KMC simulations are conducted starting from the green powder images obtained using FIB-SEM. The simulated microstructural trajectory is directly compared with the experimental observations to verify the numerical modeling. To our knowledge, the combining work, the meso-scale KMC simulations together with the measurements on real powders obtained using FIB-SEM, has not been reported yet.

\section{Experimental}

\subsection{Sample preparation}

$\mathrm{Ni}$ powder of nominal diameter $1 \mu \mathrm{m}$ was obtained from Kojundo Chemical Laboratory Co., Ltd. The impurity level of this powder was examined using glow discharge mass spectrometry by Evans Analytical Group. The analysis reveals that the purity of this $\mathrm{Ni}$ powder well exceeds 99.9 mass\%. The powder was compacted using a Newton press machine at $2 \mathrm{MPa}$. The resultant cylindrical compact, $\sim 5 \mathrm{~mm}$ diameter and $1.5 \mathrm{~mm}$ thick, was mounted onto a platinum $(\mathrm{Pt})$ pellet and sintered in an image furnace (SVF-QP2-6, Motoyama Co., Ltd). To prevent Ni oxidation, the sintering tests were performed under a reducing environment in a $1 \% \mathrm{H}_{2}$-Ar gas mixture.

Five samples with different densities were prepared using a constant-rate sintering process. The thermal histories experienced by each sample are summarized in Fig. 1. Initially, all samples were pre-sintered from room temperature to $400{ }^{\circ} \mathrm{C}$ in ten minutes. One of the five samples was analyzed at this condition (S1). The remaining four samples were continuously heated up to 600 or $750{ }^{\circ} \mathrm{C}$ at a constant heating rate of $10{ }^{\circ} \mathrm{C} / \mathrm{min}$ (S2, S4) and $40{ }^{\circ} \mathrm{C} / \mathrm{min}$ (S3, S5). The reproducibility of the samples was checked by carrying out five identical trials when preparing each of the samples (S1, S2 and S4). The standard deviations 
of the relative densities for samples $\mathrm{S} 1, \mathrm{~S} 2$ and $\mathrm{S} 4$, estimated by the mass and volume measurement, were 1.1, 1.6 and $3.8 \%$, respectively. The deviation slightly increased as the relative densities is increased, but the variations are small compared to the difference between the samples.

\subsection{D FIB-SEM reconstruction}

The microstructures of the five sintered samples were observed using 3D FIB-SEM [29]. Before observation, the samples were impregnated with epoxy resin under vacuum, which allows $\mathrm{Ni}$ and pore phases to be easily distinguished during the subsequent reconstruction process. The cured samples were polished using an argon-ion beam cross-section polisher (JEOL Ltd., SM-09010). Serial sectioning was conducted using a triple-beam FIB-SEM (SMF-1000, Hitachi High-Tech Science) for the pre-sintered sample (S1), and a dual-beam FIB-SEM (NVision40, Carl Zeiss) for the other four samples (S2-S5). The cross-sectional images, captured near the region far from the Ni/Pt pellet interface to eliminate the constraint effect of the interface on densification, were successfully collected using a $2 \mathrm{kV}$ accelerating voltage and an in-lens detector for both instruments. The resolutions of the SEM image and FIB slicing were in the ranges of $35-50 \mathrm{~nm}$ and $50-84 \mathrm{~nm}$, respectively. These resolution values are somewhat larger than the minimal resolution for FIB slicing ( $20 \mathrm{~nm}$ ) which is accessible by the current instrument (NVision40). In Table 1, the volume sizes and resolutions for five samples are shown. The volume sizes of our samples are approximately identical to the typical specimen sizes reported in the literature [30].

Figure 2 shows a typical cross-sectional 2D SEM image for the pre-sintered sample (S1) and sample (S4), where the pore and Ni phases are represented by black and gray, respectively. The boundaries between the two phases in 2D binary images were segmented by the brightness values, and the 3D topology was reconstructed by collecting the sequential $2 \mathrm{D}$ data. To eliminate subtle artifacts arising from the segmentation process, the phase boundaries were smoothed out using a filtering algorithm [37].

It is recognized that the accurate estimate of the microstructural parameters requires the appropriate resolutions for the reconstructions, which is basically dominated by the typical characteristic length; the particle size included in the system [30, 31]. Many authors have suggested that the quantity of parameters such as the surface area, volume fraction and 
the length of triple phase boundary are ensured when the resolution is below 1/10 of the particle size $[32,33]$. In this work, the averaged particle size of the powder compacts are > $0.5 \mu \mathrm{m}$, addressing that at least $50 \mathrm{~nm}$ resolution is required. In addition, it is more convenient that the subsequent simulations are conducted under the cubic voxel systems. Therefore, the 3D data was finally resampled to have a voxel size of $50 \mathrm{~nm}$ in all directions. In Figure 3, we show the obtained reconstructed microstructures for the five sintered samples.

The 3D microstructures for the five sintered compacts were quantified using the relative density $\rho$, averaged grain size $G_{i}$, averaged phase size $D_{i}$, surface to volume ratio $S_{i, \mathrm{~V}}$ and phase connectivity $\mathrm{V}_{i}$ for each phase $i$. The microstructural parameters for each compact are listed in Table 2. The relative density $\rho$ of each sample was measured as the volume fraction $\rho=N_{\mathrm{N}} / N_{\text {tot }}$, where $N_{\mathrm{N}}$ is the number of voxels corresponding to the $\mathrm{Ni}$ phase and $N_{\text {tot }}$ is the total number of voxels. As given in Table 2, the microstructures of sintered compacts over the range of $\rho=0.48-0.94$ were experimentally investigated. The surface to volume ratio $S_{i, \mathrm{~V}}$ (surface area of each phase $i$ normalized by the corresponding volume of phase $i$ ) was computed using the marching cube algorithm [34, 35]. The phase connectivity $\mathrm{V}_{i}$ for each phase was defined according to the procedure of Wilson et al. [37]. The phase size $D_{i}$ was estimated by the interception method [11], whereas the grain size $G_{i}$ was determined using a Monte Carlo as described in the next section.

\subsection{D grain generation}

The 3D information on crystal grains was numerically extrapolated into 3D reconstructed microstructures using a grain growth algorithm based on a Monte Carlo (MC) scheme [20]. Initially, a random integer number $q$ between 1 and 200 ( $q$ indicates crystallographic orientation, as explained below) was distributed on each $\mathrm{Ni}$ voxel site, and $10^{4} \mathrm{MC}$ step simulation was performed until apparent grain growth was not detected. Other parameters used in this algorithm are discussed in Section 3. Figure 4(a) shows the 3D grained Ni microstructure after extrapolation for sample S1, colored according to the $q$ value assigned to each voxel. Here, grain size $G_{i}$ was computed as the cubic root of $N_{q}$, where $N_{q}$ is the number of continuous sites having an identical value of $q$. The sizes of grains which are in contact with the outer six boundaries are likely to be underestimated than those in the interior region. 
If the distance between the center of mass and the nearest outer boundary are below $0.5 \mu \mathrm{m}$ ( typical grain size), those grains were eliminated from the grain size analysis. The number of Ni grains $N_{\mathrm{g}}$ used for averaging is provided in Table 2. By applying the same procedure to the pore phase, the grain and pore size distributions of this sample (S1) can be obtained, as shown in Fig. 4(b). Both data are exactly characterized by log-normal distributions with their peak sizes around $0.4 \mu \mathrm{m}$, indicating that our scheme obtains reasonable geometric properties of real powder compacts at the sub-micron scale. However, it should be noted that the real grain and phase shapes are considerably complicated so that the precise descriptions of the grain and phase sizes are non-trivial tasks [36]. Therefore, the value of $G_{i}$ (and $D_{i}$ ) used here would be a measure for the grain and phase sizes.

The averaged grain and pore sizes $\left(G_{i}\right.$ and $\left.D_{i}\right)$ for five reconstructed samples are shown in Table 2. The obtained sizes of $\mathrm{Ni}$ for sample (S1) are $G_{N}=0.47 \mu \mathrm{m}$ and $D_{N}=0.63 \mu \mathrm{m}$, which approximately match with the $\mathrm{Ni}$ particle size in the as-reduced Ni-YSZ system estimated by a 3D FIB-SEM technique $(0.54 \mu \mathrm{m}$ [30]). It is also found that the Ni phase size calculated by the interception method is larger than the Ni grain size from the MC method for all samples (S1-S5). Moreover, the difference between $G_{\mathrm{N}}$ and $D_{\mathrm{N}}$ becomes significant when the relative density is high (S4 and S5). Holzer et al. have pointed out in detail that the result of particle size distribution depends on the particle detection algorithm [36]. In particular, the ability to split the continuous network along the neck (grain boundary: GB) into the discrete particles critically affects the results. The MC extrapolation method has a strong advantage in splitting the neck than the interception method, because the grain growth is naturally pinned at the neck point, which produces an energetically-favorable flat GB [20]. Thus, the applied MC method would yield more realistic size distribution, while its computational cost is much higher than the interception method.

\section{Kinetic Potts Monte Carlo modeling}

The time evolution of the three-dimensional microstructure during solid-state sintering is numerically solved using a KMC method. The details of the KMC method can be found in previous studies [20, 38]. In this model, the pores and grains are described in a three-dimensional voxel system. Each voxel site $i$ has an integer number $q_{i}$ between 0 and $Q$, 
where $(Q+1)$ is the total number of possible distinct states. A unique number of $q=0$ is defined for all the pore sites, while a positive number of $q(\geq 1)$ is assigned for the grain sites, where the $q$ value characterizes the crystallographic orientation of the grains. Thus, the individual crystal grains can be identified as ensembles of continuous sites with the same positive number $q$.

In this model, sintering is driven by reducing the excess energy caused by the presence of surfaces and grain boundaries associated with a given microstructure. The excess energy $E$ of the system is described by the following equation:

$$
E=\frac{1}{2} \sum_{i=1}^{N} \sum_{j=1}^{26} J_{i j}\left(1-\delta\left(q_{i}, q_{j}\right)\right)
$$

where $N$ is the total number of sites in the system, 26 is the number of nearest-neighbor sites of site $i, J_{i j}$ is the interaction energy between sites $i$ and $j$, and $\delta$ is the Kronecker delta with $\delta=0$ for $q_{i} \neq q_{j}$ and $\delta=1$ for $q_{i}=q_{j}$. This delta function indicates that only unlike interactions between sites $i$ and $j$ can contribute to the energy $E$. Physically, $J$ corresponds to the surface energy $J_{\mathrm{s}}$ for pore-grain interaction, and the grain boundary energy $J_{\mathrm{gb}}$ for grain-grain interaction. Assuming that both $J_{\mathrm{s}}$ and $J_{\mathrm{gb}}$ are isotropic, average constant values of $J_{\mathrm{s}}=1.8 \mathrm{~J} / \mathrm{m}^{2}$ and $J_{\mathrm{gb}}=1.0 \mathrm{~J} / \mathrm{m}^{2}$ for Ni were chosen on the basis of atomistic simulation results $[39,40]$.

The kinetics of solid-state sintering are described by three mechanisms [20, 38]: (1) curvature-driven grain growth; (2) pore migration by surface diffusion; and (3) densification mediated by mass flow. During densification, vacancies (isolated pores) are formed on grain boundaries near the pore surfaces and are then diffused and annihilated along the grain boundaries. In this study, the annihilation algorithm described in Ref. [41] was adapted.

The microstructure evolves to reduce the excess energy $E$ in accordance with these kinetic mechanisms using the standard Metropolis algorithm. The parameters controlling the kinetic processes are the simulation temperatures $k_{\mathrm{B}} T$ and attempt frequencies $f$, which are chosen to match with the experimental behavior of the powder. In our study, the simulation temperatures for grain growth $k_{\mathrm{B}} T_{\mathrm{gg}}=0.5 J_{\mathrm{gb}}$, pore migration $k_{\mathrm{B}} T_{\mathrm{pm}}=1.5 J_{\mathrm{s}}$, and vacancy formation $k_{\mathrm{B}} T_{\mathrm{vf}}=13.0 J_{\mathrm{s}}$ were used. The attempt frequencies for grain growth $f_{\mathrm{gg}}=0.2$, pore 
migration $f_{\mathrm{pm}}=1.0$, and vacancy formation $f_{\mathrm{vf}}=1.0$ at one Monte Carlo step (MCS) were also applied. These sets were determined from the preliminary calculations and found to be one of the best sets. However, these sets are similar to previously reported values for $40 \mu \mathrm{m}$ copper powder simulations $[20,22]$.

Three microstructures clipped from different regions of sample (S1) were employed as starting initial structures. The simulations were conducted isothermally under free boundary conditions. Each microstructure consists of 150 voxels $\times 150$ voxels $\times 150$ voxels with a spatial resolution of $50 \mathrm{~nm}$ (cell length $L=7.5 \mu \mathrm{m}$ ). The mean and the standard deviation of each microstructural parameter for the three initial structures are listed in Table 2. The results indicate that the differences between three structures are not significant. During simulations, the microstructural data were averaged over three structures at an interval of 200-1000 MCS.

\section{Results and Discussion}

In the upper panel of Fig. 5, we show the change in 3D FIB-SEM microstructures for five samples (S1-S5). The simulated microstructural evolutions during sintering, beginning from part of the grained microstructure (S1), are shown in the lower part of Fig. 5. In the views of the KMC simulation, only the interior parts (bulk) of the powder compacts are illustrated. This bulk region is obtained by clipping the 10 grid layers ( $1 \mu \mathrm{m}$ thickness) from the six outermost surfaces. The microstructural parameters are measured for the bulk region in order to avoid surface edge effects. From Fig. 5, significant grain coarsening can be observed over the entire range of relative densities, which is a common trend in both experimental and numerical analyses. It is also noted that the representative volume of the KMC model shrinks as the relative density increases during simulations.

Figure 6 indicate the Ni grain size $G_{\mathrm{N}}$, surface to volume ratio of connected Ni phase $S_{\mathrm{N}, \mathrm{V}}$ and volume fraction of connected pore phase $V_{\mathrm{P}}$ as a function of the relative density. The red lines correspond to the case for $f_{\mathrm{gg}}=0.2, f_{\mathrm{pm}}=1.0$ and $f_{\mathrm{vf}}=1.0$, which best fits to the experimental powder behavior. The simulated microstructural parameters when the mean relative density becomes almost identical to the FIB-SEM reconstructed data are given in 
Table 2. The standard deviations of each are shown in parentheses. At low relative density (below 0.6), corresponding to the initial stage of sintering, particle contact and neck growth mainly take place, keeping the pore network open. At this stage, grain coarsening, which leads to the increase of $G_{\mathrm{N}}$ and the decrease of $S_{\mathrm{N}, \mathrm{V}}$ (Fig. 6(a, b)), can be detected. This is caused by the consumption of smaller grains by larger neighboring grains. Indeed, the real powder inherently includes many grains smaller than average size, as shown in Fig. 4(b). At the intermediate stage of sintering (relative density values between 0.6 and 0.85 ), a monotonic increase of $G_{\mathrm{N}}$ and decrease of $S_{\mathrm{N}, \mathrm{V}}($ Fig. $6(\mathrm{a}, \mathrm{b}))$ are observed. Thus, the grains coarsen as sintering proceeds. The critical feature of this stage is the aggressive formation of new grain boundaries, producing isolated pores when the relative density exceeds 0.8 (Fig. 6(c)). This pore closure is a sign of the transition to the final stage of sintering [15]. At the same time, the grain growth is accelerated (Fig. 6(a)). Finally, the grain size $G_{\mathrm{N}}$ becomes about three times larger than the initial grain size at relative density of 0.94 . Figure 7 plots the evolution of the relative density of the powder compact in MCS units. Initially, the compact shows a rapid densification up to around 0.85, and subsequently the densification rate becomes very slow. This behavior is similar to that measured in many isothermal experiments [15].

For the $f_{\mathrm{gg}}=0.2, f_{\mathrm{pm}}=1.0$ and $f_{\mathrm{vf}}=1.0$ case, shown as red lines in Fig. 6 , it is found that the KMC simulation is able to well reproduce the experimental topological changes in sub-micron powder compacts, such as Ni grain size $G_{\mathrm{N}}$, surface to volume ratio of connected Ni phase $S_{\mathrm{N}, \mathrm{V}}$ and volume fraction of connected pore phase $V_{\mathrm{P}}$, over the whole stage of sintering. This agreement also supports the idea that the physical model incorporated in the KMC simulations is capable of describing sub-micron-scale sintering. Moreover, the parameter sets are similar with the previously reported values for $40 \mu \mathrm{m}$ copper powder simulations $\left(f_{\mathrm{gg}}: f_{\mathrm{pm}}: f_{\mathrm{vf}}=1: 1: 1, k_{\mathrm{B}} T_{\mathrm{gg}} / J_{\mathrm{gb}}: k_{\mathrm{B}} T_{\mathrm{pm}} / J_{\mathrm{s}}: k_{\mathrm{B}} T_{\mathrm{vf}} / J_{\mathrm{s}}=1: 1: 13\right)[20,22]$, indicating that there would be no distinct difference of the underlying dominant kinetics between the sub-micron scale sintering and the larger scale sintering. In other words, the classical view, in which grain boundary diffusion is the primary mechanism that characterizes the densification behavior, would be acceptable at the sub-micron scale sintering.

Surface diffusion is known to be an alternative mechanism that dominates $\mathrm{Ni}$ sintering at the nanoscale $[26,27]$. Thus, clarification of the role of surface diffusion at the 
sub-micron scale is important. In the KMC model, the grain growth process is coupled with grain boundary mobility, the pore migration process is surface diffusivity and the densification process is grain boundary diffusivity. Therefore, the effect of surface diffusion on the sintering behavior can be investigated by varying the attempt frequencies of the pore migration process $f_{\mathrm{pm}}$ relative to that of the other processes $\left(f_{\mathrm{gg}}\right.$ and $\left.f_{\mathrm{vf}}\right)$ [20]. The results are shown in Fig. 6. When the pore migration frequency is reduced to $f_{\mathrm{pm}}=0.2$, grain coarsening is suppressed in the range $\rho=0.5-0.85$ (blue line), which leads to promotion of the densification rate, as shown in Fig. 7. In contrast, if the pore migration frequency is elevated to $f_{\mathrm{pm}}=5.0$, grain coarsening is enhanced during sintering (green line), giving a slower densification rate (Fig. 7). In addition, the case in which only pore migration and grain growth processes are operative was also examined as a special situation $\left(f_{\mathrm{gg}}=0.2, f_{\mathrm{pm}}=1.0\right.$ and $f_{\mathrm{vf}}=0.0$ ). The evolution of $\rho, G_{\mathrm{N}}$, and $S_{\mathrm{N}, \mathrm{V}}$ (dotted line in Figs. 6 and 7 ) clearly demonstrates that surface diffusion does not make any contribution to the densification of the powder compacts, but contributes only to the coarsening. Thus, surface diffusion has an impact on the microstructural evolution during sub-micron-scale sintering, acting as one of the multiple mechanisms of sintering, while surface diffusion itself is not a major mechanism of densification.

The microstructural changes predicted numerically under isothermal conditions match well with the results of the non-isothermal experiments. Su et al. demonstrated that this consistency can be achieved when the theory of a master sintering curve is established [42]. Although this theory is powerful for describing the densification process [28, 43], its applicability relies on two basic assumptions: first, that microstructural evolution is only a function of the relative density, and second, that one diffusion mechanism dominates the densification process at a given temperature range. When these assumptions are valid, the sintering path, such as the grain size-density trajectory, for the given powder compacts becomes unique regardless of their thermal histories. The path consistency shown in our results implies that the above assumptions would be reasonable for the Ni sintering process at the sub-micron scale.

Finally, we studied the effect of simulation cell size $L$ on the whole sintering behavior. In particular, as shown in Table 2 and Fig. 5, the number of grains $N_{\mathrm{g}}$ included in the system considerably decrease during sintering due to the grain coarsening. This may lead 
to the poor grain-size statistics at higher density $(>0.85)$. Here, the simulation with larger cell size $L=15 \mu \mathrm{m}$ consisting of 300 voxels $\times 300$ voxels $\times 300$ voxels was examined for the $f_{\mathrm{gg}}$ $=0.2, f_{\mathrm{pm}}=1.0$ and $f_{\mathrm{vf}}=1.0$ case. In Table 2 , we have summarized the microstructural parameters for the larger cell size. Compared with the FIB-SEM data, the deviations of Ni grain size $G_{\mathrm{N}}$ for $\rho=0.59,0.81$, and 0.94 were only about $3.2 \%, 2.8 \%$, and $0.5 \%$, respectively. This excellent agreement for the wide range of the relative density implies that the cell size for both the FIB-SEM data and the larger computational model would be reasonable to obtain the proper grain-size statistics, even at high density. On the other hand, the deviations of Ni grain size $G_{\mathrm{N}}$ between the $L=7.5 \mu \mathrm{m}(150 \times 150 \times 150$ voxels $)$ cell and $L=$ $15 \mu \mathrm{m}(300 \times 300 \times 300$ voxels $)$ cell for $\rho=0.59,0.81$, and 0.94 were about $1.6 \%, 5.5 \%$, and $10.4 \%$, showing that the deviation increases with the relative density. This would be attributed to the statistical error associated with the grain size averaging in different cell size. As an alternative evidence, as shown in Table 2, the standard deviations of the microstructural parameters among the three samples with small cell size become larger with the relative density. However, in the present study, the cell size effect seems to be significantly suppressed by using three samples for statistical averaging.

\section{Conclusions}

An effective framework to develop a numerical tool that is able to predict the microstructural evolution during sub-micron-scale sintering is developed. The framework consists of two parts. First, the 3D topological change during sintering is quantitatively characterized using FIB-SEM technique, which has an appropriate resolution to reconstruct the particle systems with the size around $0.5 \mu \mathrm{m}$. Second, the microstructural evolution, initiated from the real powder structure, was simulated using KMC modeling, and the obtained results are directly verified using the FIB-SEM assessments. In this study, the framework was applied to single-phase Ni sintering. The results successfully demonstrate that KMC simulation has the ability to reproduce the true microstructural evolution over an entire sintering stage of sub-micron powder compacts, further elucidating the underlying kinetic mechanism at this scale. This framework can be extended to more complex systems such as mixed-powder sintering at the sub-micron scale. The predictive tool for this process will be highly effective for designing electrode microstructures of solid oxide fuel cells. For instance, 
the sintered microstructures can be controlled by numerically tuning the numerous parameters associated with the starting powder microstructure, such as volume fractions, grain sizes and distributions of the two phases. Co-sintering experiment and KMC simulation of NiO-YSZ composite system which is the common SOFC anode starting material are in progress as an ongoing work.

\section{Acknowledgement}

The authors acknowledge financial support for this work through Japan Science and Technology Agency (JST) CREST program.

\section{References}

[1] A. Atkinson, S. Barnett, R.J. Gorte, J.T.S. Irvine, A.J. McEvoy, M. Mogensen, S.C. Singhal, J. Vohs, Nat. Mater. 3 (2004) 17-27.

[2] R.M. Ormerod, Chem. Soc. Rev. 32 (2003) 17-28.

[3] P.R. Shearing, D.J.L. Brett, N.P. Brandon, Int. Mater. Rev. 55 (2010) 347-363.

[4] N. Shikazono, Y. Sakamoto, Y. Yamaguchi, N. Kasagi, J. Power Sources 193 (2009) $530-540$.

[5] T. Fukui, S. Ohara, M. Naito, K. Nogi, J. Power Sources 110 (2002) 91-95.

[6] J.R. Wilson, J.S. Cronin, S.A. Barnett, Scr. Mater. 65 (2011) 67-72.

[7] H. Ohrui, T. Matsushima, T. Hirai, J. Power Sources 71 (1998) 185-189.

[8] Z. Jiao, G. Lee, N. Shikazono, N. Kasagi, J. Electrochem. Soc. 159 (2012) F278-286.

[9] H. Yokokawa, K. Yamaji, M.E. Brito, H. Kishimoto, T. Horita, J. Power Sources 196 (2011) 7070-7075.

[10] L. Holzer, B. Münch, B. Iwanschitz, M. Cantoni, T. Hocker, T. Graule, J. Power Sources 196 (2011) 7076-7089.

[11] A. Faes, D. Presvytes, C.G. Vayenas, J. Van, Fuel Cells (2009) 841-851.

[12] G.J. Nelson, K.N. Grew, J.R. Izzo, J.J. Lombardo, W.M. Harris, A. Faes, A. Hessler-Wyser, J. Van herle, S. Wang, Y.S. Chu, A. V. Virkar, W.K.S. Chiu, Acta Mater. 60 (2012) 3491-3500.

[13] K.N. Grew, W.K.S. Chiu, J. Power Sources 199 (2012) 1-13. 
[14] Y.-H. Lee, H. Muroyama, T. Matsui, K. Eguchi, J. Power Sources 262 (2014) 451-456.

[15] R.M. German, Crit. Rev. Solid State Mater. Sci. 35 (2010) 263-305.

[16] M.W. Reiterer, K.G. Ewsuk, J. Am. Ceram. Soc. 92 (2009) 1419-1427.

[17] F. Wakai, K.A. Brakke, Acta Mater. 59 (2011) 5379-5387.

[18] Y. Wang, Acta Mater. 54 (2006) 953-961.

[19] C.L. Martin, R.K. Bordia, Acta Mater. 57 (2009) 549-558.

[20] V. Tikare, M. Braginsky, D. Bouvard, A. Vagnon, Comput. Mater. Sci. 48 (2010) 317-325.

[21] C.G. Cardona, V. Tikare, B.R. Patterson, E. Olevsky, J. Am. Ceram. Soc. 95 (2012) 2372-2382.

[22] R. Bjørk, V. Tikare, H.L. Frandsen, N. Pryds, Scr. Mater. 67 (2012) 81-84.

[23] O. Lame, D. Bellet, M. Di Michiel, D. Bouvard, Acta Mater. 52 (2004) 977-984.

[24] P. Schneider, M. Meier, R. Wepf, R. Müller, Bone 49 (2011) 304.

[25] E. Maire, P.J. Withers, Int. Mater. Rev. 59 (2014) 1-43.

[26] S. Tsyganov, J. Kästner, B. Rellinghaus, T. Kauffeldt, F. Westerhoff, D. Wolf, Phys. Rev. B 75 (2007) 1-9.

[27] B.B. Panigrahi, Mater. Sci. Eng. A 460-461 (2007) 7-13.

[28] D.C. Blaine, S.J. Park, P. Suri, R.M. German, Metall. Mater. Trans. A 37 (2006) 2827-2835.

[29] H. Iwai, N. Shikazono, T. Matsui, H. Teshima, M. Kishimoto, R. Kishida, D. Hayashi, K. Matsuzaki, D. Kanno, M. Saito, H. Muroyama, K. Eguchi, N. Kasagi, H. Yoshida, J. Power Sources 195 (2010) 955.

[30] J. Joos, M. Ender, I. Rotscholl, N.H. Menzler, E. Ivers-Tiffée, J. Power Sources 246 (2014) 819-830.

[31] L. Holzer, F. Indutnyi, P.H. Gasser, B. Munch, M. Wegmann, J. Microsc. , 216 (2004) 84-95.

[32] J. Joos, M. Ender, T. Carraro, A. Weber, E. Ivers-Tiffée, Electrochim. Acta 82 (2012) 268-276.

[33] N. Shikazono, D. Kanno, K. Matsuzaki, H. Teshima, S. Sumino, N. Kasagi, J. Electrochem. Soc. 157 (2010) B665-672.

[34] W.E. Lorensen, H.E. Cline, Comput. Graph. 21 (1987) 163-169. 
[35] K. Matsuzaki, N. Shikazono, N. Kasagi, J. Power Sources 196 (2011) 3073-3082.

[36] L. Holzer, B. Iwanschitz, T. Hocker, B. Münch, M. Prestat, D. Wiedenmann, J. Power Sources 196 (2011) 1279-1294.

[37] J.R. Wilson, M. Gameiro, K. Mischaikow, W. Kalies, P.W. Voorhees, S.A. Barnett, Microsc. Microanal. 15 (2009) 71-77.

[38] M. Braginsky, V. Tikare, E. Olevsky, Int. J. Solids Struct. 42 (2005) 621-636.

[39] Y. Mishin, D. Farkas, M. Mehl, D. Papaconstantopoulos, Phys. Rev. B 59 (1999) 3393-3407.

[40] D.L. Olmsted, E.A. Holm, S.M. Foiles, Acta Mater. 57 (2009) 3704-3713.

[41] R. Bjørk, H.L. Frandsen, V. Tikare, E. Olevsky, N. Pryds, Comput. Mater. Sci. 82 (2014) 293-297.

[42] H. Su, D.L. Johnson, J. Am. Ceram. Soc. 12 (1996) 3211-3217.

[43] K.G. Ewsuk, D.T. Ellerby, C.B. DiAntonio, J. Am. Ceram. Soc. 89 (2006) 2003-2009. 
Table 1 Volume size and resolution of 3D FIB-SEM data for five sintered compacts (S1-S5).

\begin{tabular}{cccccc}
\hline Sample & S1 & S2 & S3 & S4 & S5 \\
\hline \hline Volume $\left[\mu \mathrm{m}^{3}\right]$ & 3375 & 1215 & 911 & 940 & 922 \\
\hline Pixel size $[\mathrm{nm}]$ & 50.0 & 40.2 & 34.8 & 40.3 & 40.2 \\
\hline Image distance $[\mathrm{nm}]$ & 50.0 & 84.2 & 84.2 & 63.4 & 65.1 \\
\hline
\end{tabular}


Table 2 The microstructural parameters measured from FIB-SEM reconstructed data for five sintered compacts (S1-S5). KMC simulation results for both $L=7.5 \mu \mathrm{m}$ and $15.0 \mu \mathrm{m}$ models are also shown for comparison, which correspond to the case for $f_{\mathrm{gg}}=0.2, f_{\mathrm{pm}}=1.0$ and $f_{\mathrm{vf}}=1.0$. The results of $L=7.5 \mu \mathrm{m}$ model present the mean values and standard deviations (parentheses) for three configurations.

\begin{tabular}{|c|c|c|c|c|c|c|}
\hline \multirow{3}{*}{$\begin{array}{l}\text { Microstructural } \\
\text { parameters }\end{array}$} & Exp. & $\mathrm{S} 1$ & S2 & S3 & S4 & S5 \\
\hline & Sim. & \multicolumn{5}{|c|}{$L=7.5[\mu \mathrm{m}]$} \\
\hline & \multicolumn{3}{|l|}{ Sim. } & \multicolumn{3}{|l|}{$L=15.0[\mu \mathrm{m}]$} \\
\hline \multirow{3}{*}{ Relative density: $\rho$} & Exp. & 0.48 & 0.56 & 0.59 & 0.81 & 0.94 \\
\hline & Sim. & $0.48(0.00)$ & $0.56(0.01)$ & $0.59(0.02)$ & $0.81(0.03)$ & $0.94(0.02)$ \\
\hline & Sim. & 0.48 & 0.56 & 0.59 & 0.81 & 0.94 \\
\hline \multirow{3}{*}{ The number of Ni grains: } & Exp. & 8286 & 1192 & 872 & 272 & 80 \\
\hline & Sim. & $820(51)$ & $278(13)$ & $213(9)$ & $44(5)$ & $20(4)$ \\
\hline & Sim. & 8286 & 3559 & 2796 & 625 & 131 \\
\hline \multirow{3}{*}{ Averaged Ni grain size: } & Exp. & 0.47 & 0.65 & 0.65 & 1.12 & 1.94 \\
\hline & Sim. & $0.47(0.01)$ & $0.58(0.01)$ & $0.64(0.01)$ & $1.15(0.07)$ & $1.73(0.15)$ \\
\hline & Sim. & 0.47 & 0.57 & 0.63 & 1.09 & 1.93 \\
\hline \multirow{2}{*}{ Averaged Ni phase size: } & Exp. & 0.63 & 0.85 & 0.86 & 1.74 & 4.03 \\
\hline & Sim. & $0.61(0.02)$ & $0.78(0.01)$ & $0.86(0.01)$ & $1.84(0.14)$ & $3.36(0.33)$ \\
\hline$D_{\mathrm{N}}[\mu \mathrm{m}]$ & Sim. & 0.63 & 0.81 & 0.90 & 2.22 & 5.52 \\
\hline \multirow{2}{*}{ Averaged pore size: } & Exp. & 0.55 & 0.60 & 0.54 & 0.51 & 0.49 \\
\hline & Sim. & $0.55(0.01)$ & $0.57(0.03)$ & $0.60(0.02)$ & $0.65(0.05)$ & $0.72(0.39)$ \\
\hline$G_{\mathrm{P}}[\mu \mathrm{m}]$ & Sim. & 0.55 & 0.51 & 0.55 & 0.68 & 0.77 \\
\hline \multirow[t]{2}{*}{ Averaged pore phase size: } & Exp. & 0.65 & 0.73 & 0.68 & 0.77 & 0.99 \\
\hline & Sim. & $0.62(0.02)$ & $0.68(0.03)$ & $0.71(0.03)$ & $0.87(0.05)$ & $0.81(0.12)$ \\
\hline$D_{\mathrm{P}}[\mu \mathrm{m}]$ & Sim. & 0.65 & 0.69 & 0.73 & 1.05 & 0.97 \\
\hline \multirow{3}{*}{$\begin{array}{l}\text { Surface to volume ratio of } \\
\text { connected Ni: } S_{\mathrm{N}, \mathrm{V}}\left[\mu \mathrm{m}^{-1}\right]\end{array}$} & Exp. & 6.56 & 4.46 & 4.34 & 1.73 & 0.46 \\
\hline & Sim. & $6.47(0.15)$ & $4.52(0.08)$ & $3.94(0.09)$ & $1.29(0.15)$ & $0.39(0.08)$ \\
\hline & Sim. & 6.56 & 4.62 & 4.03 & 1.34 & 0.40 \\
\hline \multirow{3}{*}{$\begin{array}{l}\text { Surface to volume ratio of } \\
\text { connected pore: } S_{\mathrm{P}, \mathrm{V}}\left[\mu \mathrm{m}^{-1}\right]\end{array}$} & Exp. & 6.23 & 5.75 & 6.54 & 8.09 & 8.33 \\
\hline & Sim. & $6.24(0.24)$ & $5.72(0.33)$ & $5.61(0.34)$ & $5.50(0.70)$ & $5.69(0.84)$ \\
\hline & Sim. & 6.23 & 5.98 & 5.86 & 5.55 & 5.69 \\
\hline \multirow{3}{*}{$\begin{array}{l}\text { Volume fraction of } \\
\text { connected } \mathrm{Ni}: V_{\mathrm{N}}\end{array}$} & Exp. & 1.00 & 1.00 & 1.00 & 1.00 & 1.00 \\
\hline & Sim. & $1.00(0.00)$ & $1.00(0.00)$ & $1.00(0.00)$ & $1.00(0.00)$ & $1.00(0.00)$ \\
\hline & Sim. & 1.00 & 1.00 & 1.00 & 1.00 & 1.00 \\
\hline \multirow{3}{*}{$\begin{array}{l}\text { Volume fraction of } \\
\text { connected pore: } V_{\mathrm{P}}\end{array}$} & Exp. & 1.00 & 1.00 & 1.00 & 0.98 & 0.61 \\
\hline & Sim. & $1.00(0.00)$ & $1.00(0.00)$ & $1.00(0.00)$ & $0.94(0.06)$ & $0.85(0.12)$ \\
\hline & Sim. & 1.00 & 1.00 & 1.00 & 0.98 & 0.50 \\
\hline
\end{tabular}




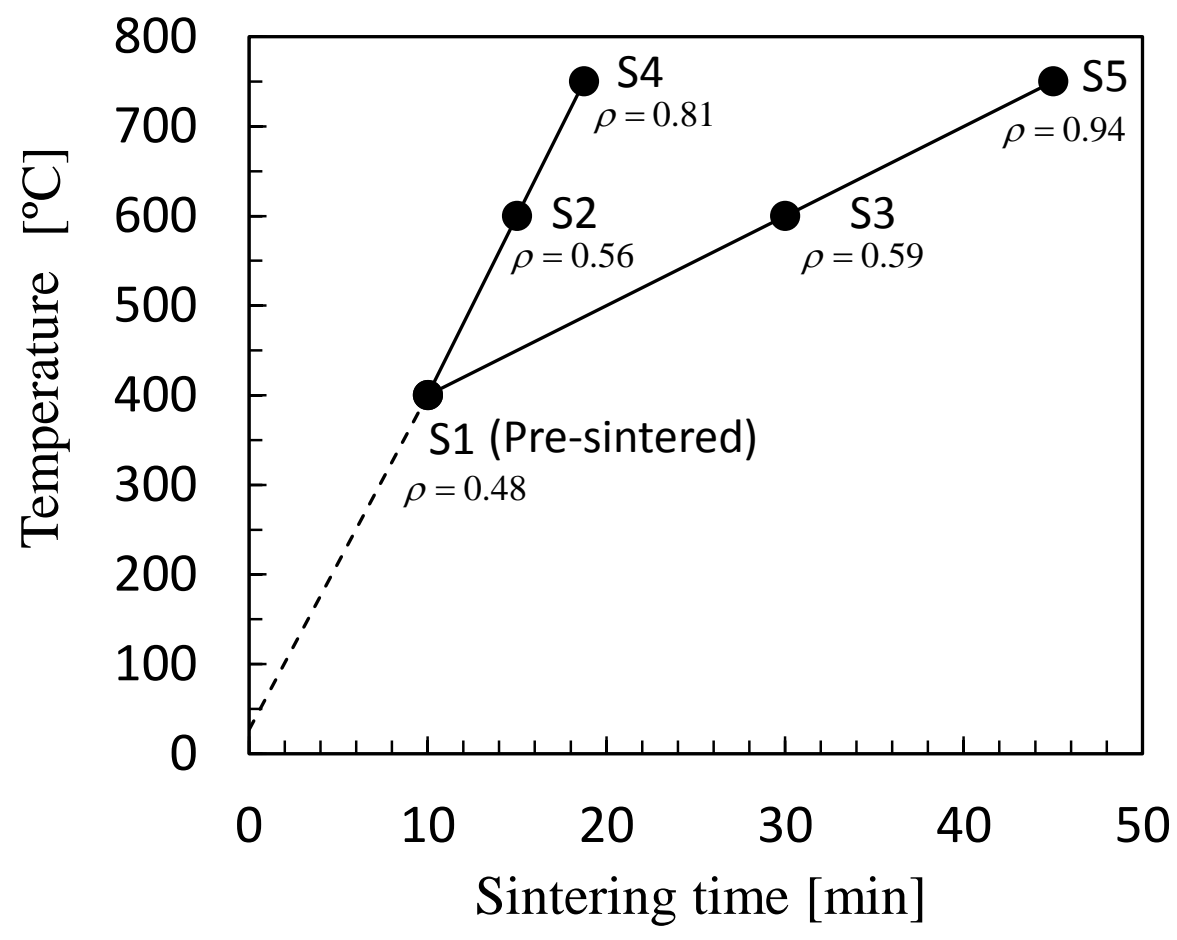

Fig. 1 Non-isothermal treatments applied to the five Ni samples (S1-S5). The corresponding relative densities of the samples, measured from the 3D FIB-SEM reconstructed structures, are also shown. 
(a) $9.0 \mu \mathrm{m}$

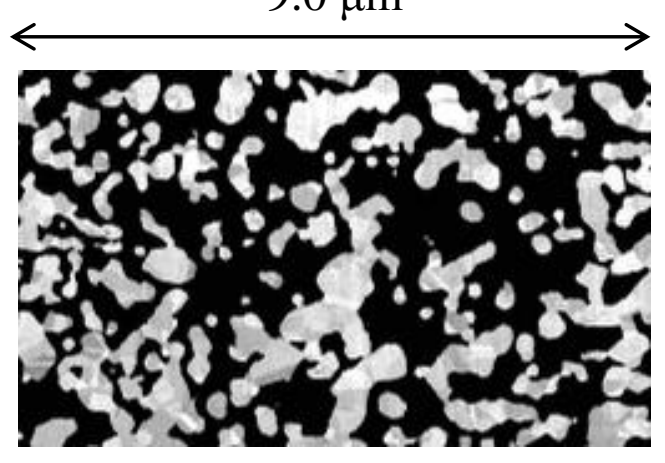

(b)

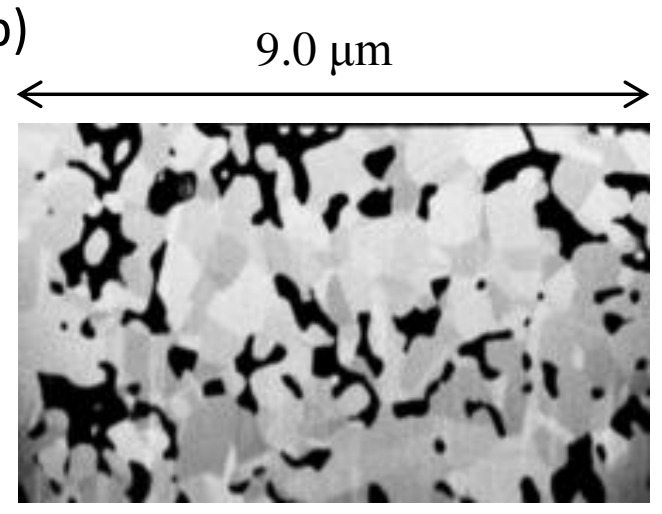

Fig. 2 2D SEM cross-sectional views before reconstruction (Ni phase: gray, pore phase: black) sintered up to relative densities of (a) 0.48 (sample: S1) and (b) 0.81 (sample: S4). 

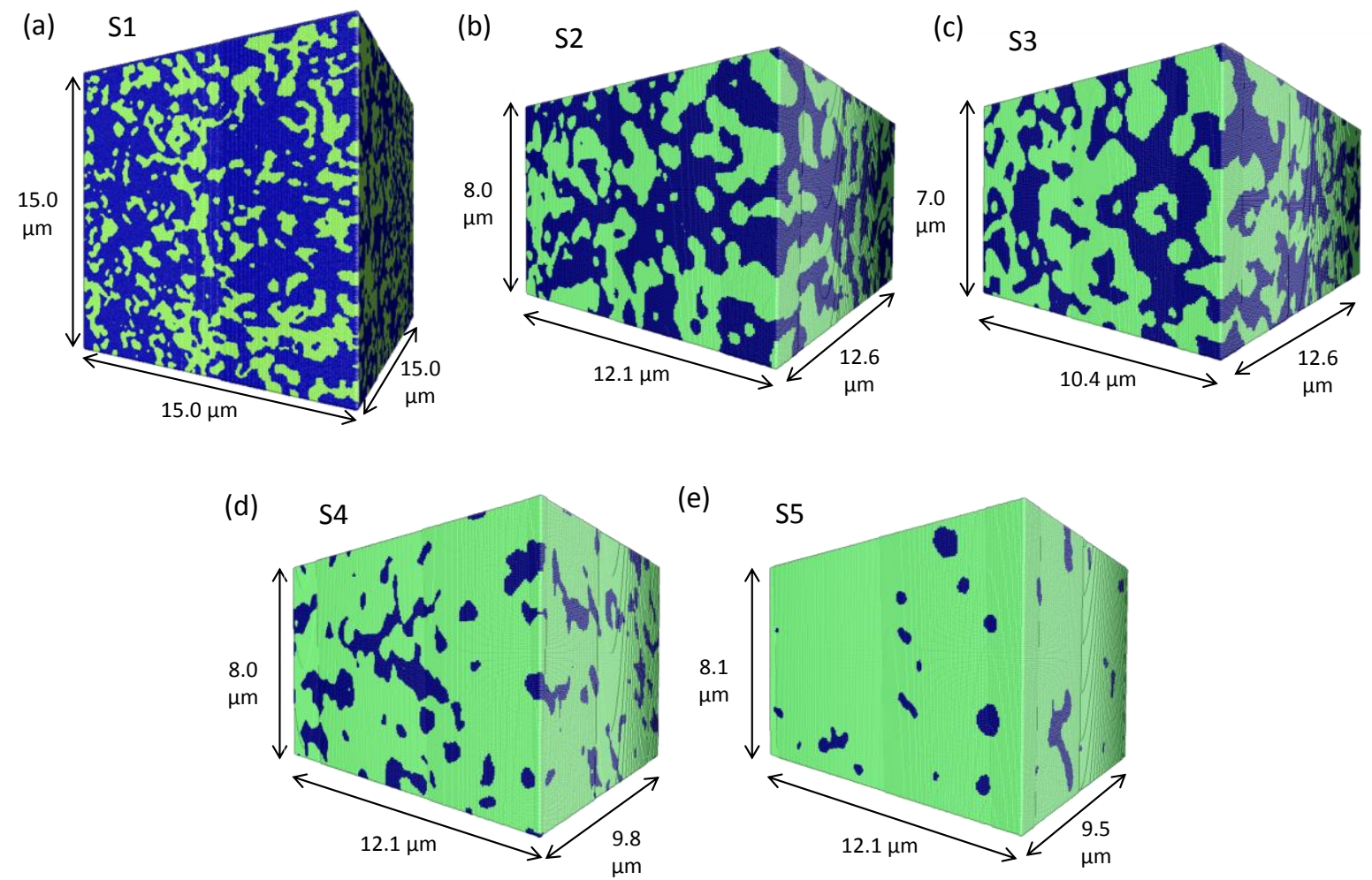

Fig. 3 3D FIB-SEM reconstructed microstructures, resampled to have a resolution of $50 \mathrm{~nm}$ in all directions (Ni phase: green, pore phase: blue) with the relative densities of (b) 0.48 (sample: S1), (c) 0.56 (sample: S2), (d) 0.59 (sample: S3), (e) 0.81 (sample: S4) and (f) 0.94 (sample: S5). 

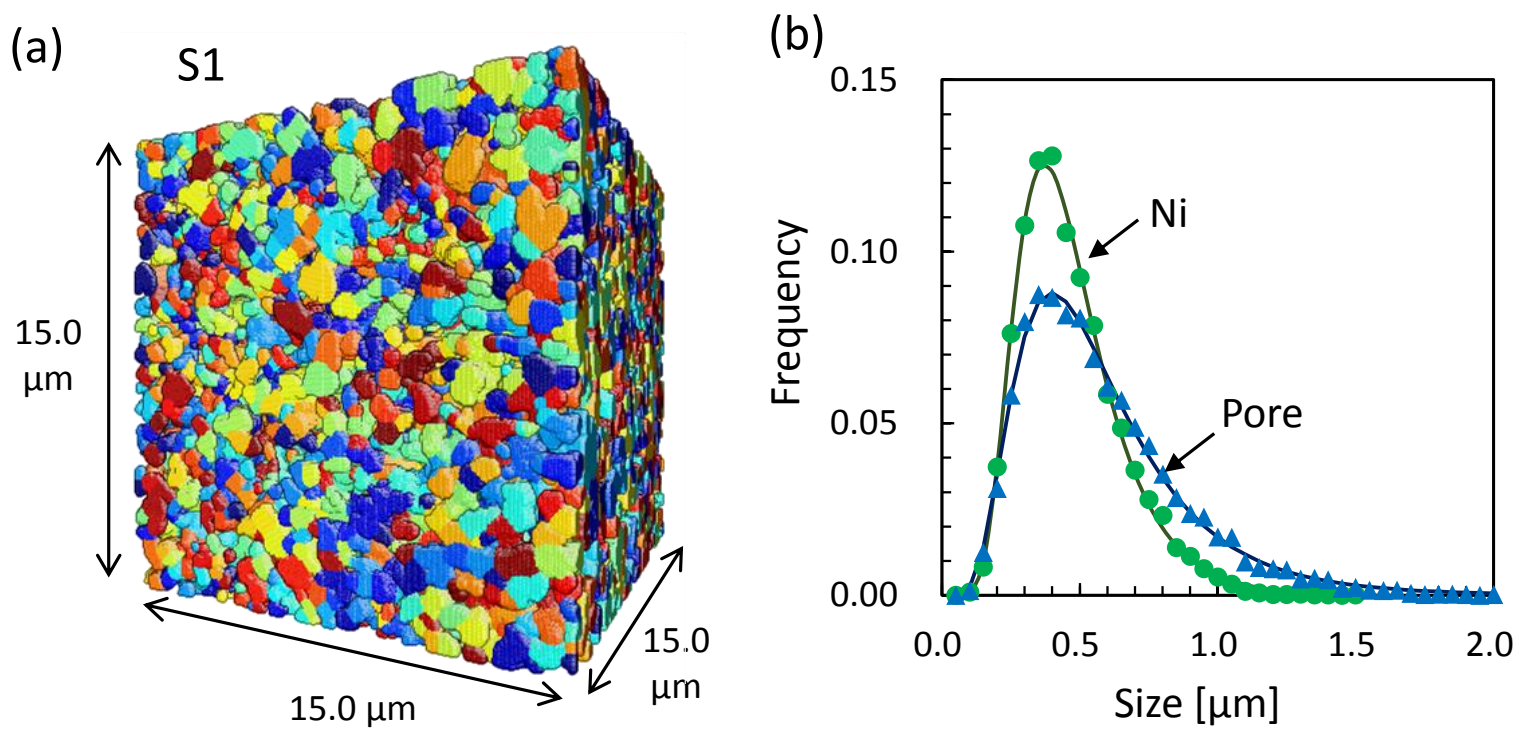

Fig. 4 Reconstructed starting microstructure for KMC simulation, which corresponds to the Ni powder compact (sample: S1) sintered up to relative density of 0.48 . (a) 3D grained $\mathrm{Ni}$ microstructure, of which the sub-micron sized grains are generated into the 3D FIB-SEM data using grain growth algorithm. (b) Ni grain and pore size distributions with lognormal fit. 


\section{D FIB-SEM reconstruction}

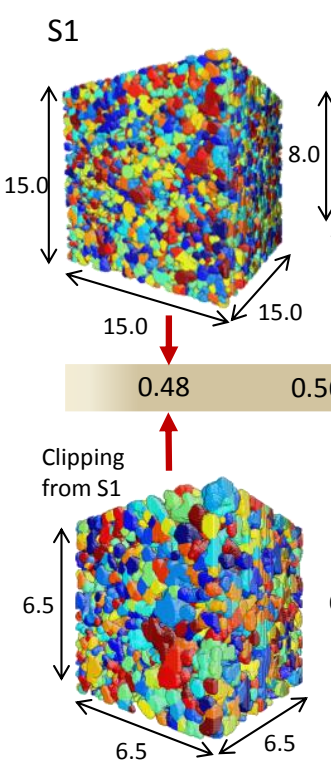

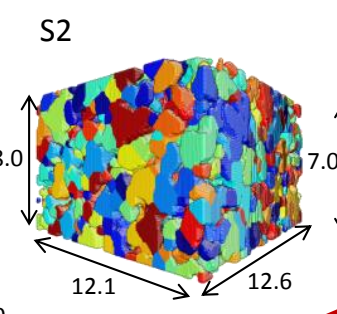

S3

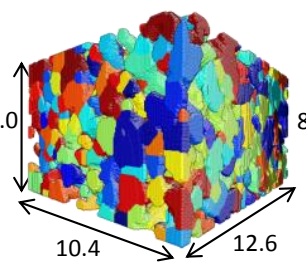

S4

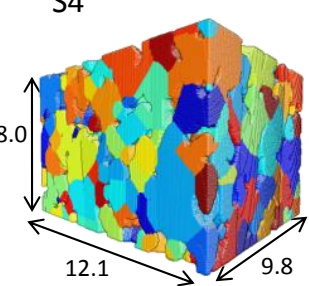

S5

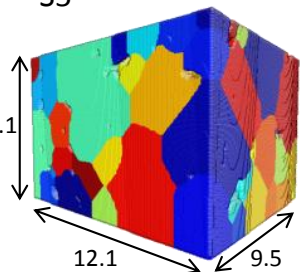

$\downarrow$

$0.94 \quad 1.0$

Relative
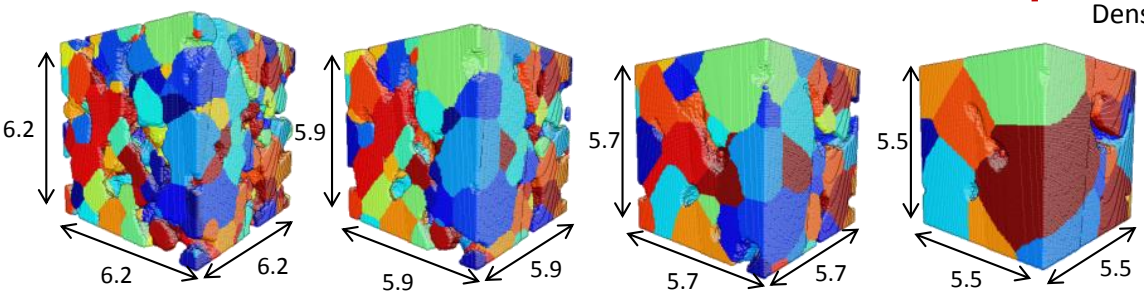

3D kinetic Monte Carlo

Fig. 5. Change in microstructures of powder compacts obtained from (upper) 3D FIB-SEM image analysis for five samples (S1-S5) after the numerical generation of the grain structure, and (lower) 3D KMC simulations, started from a part of the grained microstructure (S1). Simulation models are illustrated only for the interior part (bulk) of the powder compacts, where the microstructural parameters are computed. The cell length is written in unit of micrometer. 

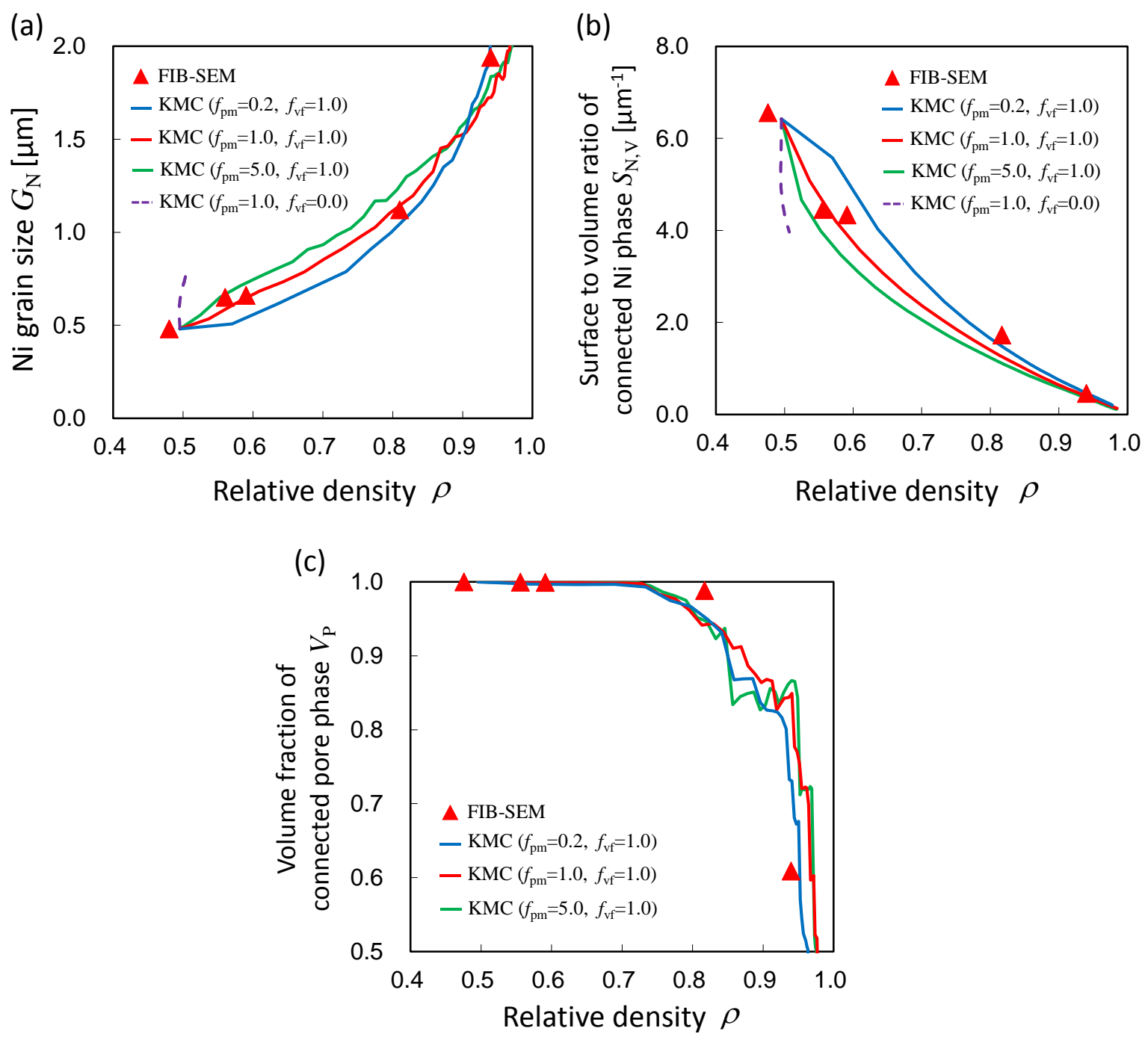

Fig. 6 Evolution of microstructural parameters; (a) Ni grain size $G_{\mathrm{N}}$, (b) surface to volume ratio of connected $\mathrm{Ni}$ phase $S_{\mathrm{N}, \mathrm{V}}$ and (c) volume fraction of connected pore phase $V_{\mathrm{P}}$ as a function of relative density of powder compacts. Simulation results for different attempt frequency of pore migration $f_{\mathrm{pm}}$ and vacancy formation $f_{\mathrm{vf}}$ are presented. For all cases, the attempt frequency of grain growth $f_{\mathrm{gg}}$ is set to be 0.2. For comparison, the experimental values (FIB-SEM) are also plotted. 


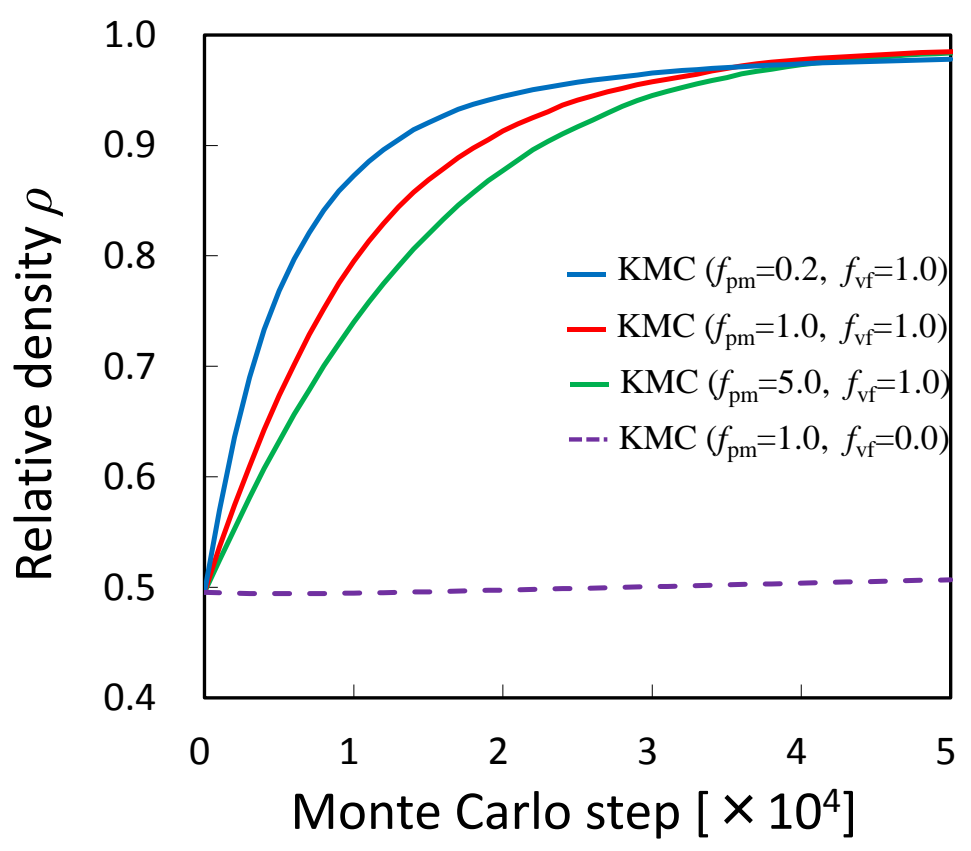

Fig. 7 Densification curves during isothermal sintering simulations for different attempt frequency of pore migration $f_{\mathrm{pm}}$ and vacancy formation $f_{\mathrm{vf}}$. For all cases, the attempt frequency of grain growth $f_{\mathrm{gg}}$ is set to be 0.2 . 